\title{
The impact of main belt asteroids on infrared-submillimetre photometry and source counts (Research Note)
}

\author{
Cs. Kiss ${ }^{1}$, A. Pál ${ }^{2}$, T. G. Müller ${ }^{3}$, and P. Ábrahám ${ }^{1}$ \\ 1 Konkoly Observatory of the Hungarian Academy of Sciences, PO Box 67, 1525 Budapest, Hungary \\ e-mail: pkisscs@konkoly.hu \\ 2 Department of Astronomy, Eötvös University, Pázmány Péter st. 1/A, 1117 Budapest, Hungary \\ 3 Max-Planck-Institut für extraterrestrische Physik, Giessenbachstrasse, 85748 Garching, Germany
}

Received 29 August 2007 / Accepted 19 November 2007

\begin{abstract}
Context. Among the components of the infrared and submillimetre sky background, the closest layer is the thermal emission of dust particles and minor bodies in the Solar System. This contribution is especially important for current and future infrared and submillimetre space instruments - like those of Spitzer, Akari and Herschel - and must be characterised by a reliable statistical model.

Aims. We describe the impact of the thermal emission of main belt asteroids on the $5 \ldots 1000 \mu \mathrm{m}$ photometry and source counts, for the current and future spaceborne and ground-based instruments, in general, as well as for specific dates and sky positions.

Methods. We used the statistical asteroid model (SAM) to calculate the positions of main belt asteroids down to a size of $1 \mathrm{~km}$, and calculated their infrared and submillimetre brightness using the standard thermal model. Fluctuation powers, confusion noise values and number counts were derived from the fluxes of individual asteroids.

Results. We have constructed a large database of infrared and submillimetre fluxes for SAM asteroids with a temporal resolution of 5 days, covering the time span January 1, 2000-December 31, 2012. Asteroid fluctuation powers and number counts derived from this database can be obtained for a specific observation setup via our public web-interface.

Conclusions. Current space instruments working in the mid-infrared regime (Akari and Spitzer Space Telescopes) are affected by asteroid confusion noise in some specific areas of the sky, while the photometry of space infrared and submillimetre instruments in the near future (e.g. Herschel and Planck Space Observatories) will not be affected by asteroids. Faint main belt asteroids might also be responsible for most of the zodiacal emission fluctuations near the ecliptic.
\end{abstract}

Key words. radiation mechanisms: thermal - astronomical data bases: miscellaneous - infrared: solar system minor planets, asteroids

\section{Introduction}

Due to their relatively high apparent brightness at infrared wavelength compared e.g. to Galactic stars, asteroids are among the dominant sources at infrared wavelengths and can seriously affect the infrared and submillimetre photometry and source counts. Currently, 377328 minor planets are known ${ }^{1}$ (as of July 30, 2007) in our Solar System, of which about $99 \%$ are located in the main belt. On the plane of the sky the vast majority of main belt asteroids (MBAs) are found at ecliptic latitudes below $20^{\circ}$. They have sizes between a few ten meters up to about $1000 \mathrm{~km}$. With temperatures between 200 and $300 \mathrm{~K}$, the asteroids emit predominantly at thermal wavelengths between $5 \mu \mathrm{m}$ and the millimetre range. Deep infrared observations close to the ecliptic will therefore always include some of these moving targets (e.g. Tedesco \& Désert 2002; Meadows et al. 2004). Such observations also show that only a small fraction of the existing minor body population is currently known and this population might cause a non-negligible confusion noise contribution at certain wavelengths and for specific instruments.

\footnotetext{
${ }^{1}$ http://cfa-www.harvard.edu/iau/lists/ ArchiveStatistics.html
}

Tedesco \& Désert (2002) measured the number of main belt asteroids for the first time in a direct way by using the Infrared Space Observatory (ISO). Based on a statistical asteroid model the authors concluded that there are about $1.2 \pm 0.5 \times 10^{6}$ asteroids ( $\geq 1 \mathrm{~km}$ in diameter) in our solar system, twice as many as previously believed.

A set of celestial sources may affect compact source observations in two ways: (1) they contribute to the confusion noise, the uncertainty in point source photometry due to the fluctuations of the sky background; and (2) they also appear as individual sources, that may add "false" detections to source counts.

Recently, several authors calculated confusion noise and detection limits for current/future infrared space missions (Spitzer, Akari, Herschel and SPICA). These papers considered the two major confusion noise components: the extragalactic background (Lagache et al. 2003; Negrello et al. 2004), the Galactic cirrus emission (Kiss et al. 2005; Jeong et al. 2005), or the combination of the two (Jeong et al. 2006).

It is an important question for infrared space missions whether faint asteroids close to or below the detection limit could contribute significantly to the confusion noise of these instruments or could be present as a significant count of 
contaminating point sources in the field of view. To consider these asteroids, a reliable statistical model is needed. Recently, Tedesco et al. (2005) presented the "Statistical Asteroid Model" (hereafter SAM). This model is based on a population of $\sim 1.9 \times$ $10^{6}$ asteroids obtained from the complete known asteroid sample (as of 1999), plus extrapolation of the size-frequency distribution (SFD) of 15 asteroid dynamical families and three background populations, to a diameter limit of $1 \mathrm{~km}$. The validity of the SAM was demonstrated by comparing SAM predictions with ISO measurements at $12 \mu \mathrm{m}$ (Tedesco \& Désert 2002) and Spitzer measurements at the 8 and $24 \mu \mathrm{m}$ photometic bands (Meadows et al. 2004). The asteroid counts from both deep surveys show good agreement with the SAM predictions.

In this paper we give (1) estimates for the impact of MBAs on infrared (IR) to submillimetre (especially spaceborne) measurements, such as asteroid number counts and confusion noise, for a specific date, sky position, wavelength/instrument; and (2) observable predictions for the infrared regime which can be used to check the parameters of the present model, e.g. size, albedo and positional distribution. This is the first study that investigates the impact of asteroids on infrared and submillimetre photometry and source counts in detail, based on a realistic asteroid sample.

\section{Data processing}

\subsection{Position calculation}

Orbital elements of all SAM asteroids, including the real and predicted ones were calculated for different epochs between the time span January 1, 2000 and December 31, 2012 with an average step size of 5 days. This step size is fine for yielding a good coverage of the solar elongation in a year. The orbital elements were obtained using accurate numerical integrations including the effect of all inner and outer planets. From the orbital parameters the apparent ecliptic coordinates, distances and magnitudes were derived using the spatial coordinates of the Earth itself and the absolute magnitudes (which is known from the SAM database). Some of the spacecrafts, including the Herschel and Planck Space Observatories will be located at the L2-point of the Earth-Moon system. The difference between the spatial position of the barycentre of the Earth-Moon system and the prospective position of the L2-point is negligible for the apparent distribution of the asteroids. However, the Spitzer Space Telescope is in a significant distance from Earth, therefore we performed the position and all subsequent other calculations for the Spitzer Space Telescope coordinates for a limited time span, covering the expected cryogenic lifetime between January 1, 2004 and December 31, 2009. The actual positions of the Spitzer Space Telescope were taken from the NASA/JPL HORIZONS system $^{2}$.

To test the positional accuracy of our integration, we compared the observable ephemerides yielded by the integration and the coordinates returned by the Minor Planet \& Comet Ephemeris Service of the Harvard-Smithsonian Center for Astrophysics $^{3}$ for a couple of known minor and dwarf planets, including Ceres, Pallas, Vesta and Astraea. The comparison timespan was almost 105 years, resulted by a backward integration from March 6, 2006 to 1901.0. The differences between the two sets were always smaller than 0.04 for a specific minor planet. We have also compared this difference for a timespan

\footnotetext{
2 http://ssd.jpl.nasa.gov/?horizons

3 http://www. cfa.harvard.edu/iau/MPEph/MPEph.html
}

of 12 years, the same as the maximal integration period of the complete simulation. In this case the error was definitely smaller than 0.004 .

\subsection{Thermal brightness calculation}

For each SAM asteroid and for each date thermal fluxes were assigned at 14 fixed wavelengths $\left\{\lambda_{0, k}\right\}$. These wavelengths were chosen the cover the $5 \mu \mathrm{m}$ to $1 \mathrm{~mm}$ range in a logarithmically equidistant way. If a $\lambda_{i}$ wavelength was different from $\left\{\lambda_{0, k}\right\}$, the monochromatic flux values were interpolated to the desired $\lambda_{i}$ for each asteroid, individually. For the brightness calculations we applied the Standard Thermal Model (STM, see Lebofsky et al. 1986). In this model the surface temperature distribution is calculated using the true heliocentric and geocentric distances. The asteroids are described as smooth, spherical and non-rotating bodies in instantaneous equilibrium with the solar radiation. No heat conduction into the surface is considered. The correction for beaming, shape and conductivity effects is done via the $\eta$-parameter with a value of $\eta=0.756$. Furthermore, the flux at non-zero solar phase angles is obtained by applying an empirical phase correction of $0.01 \mathrm{mag} \mathrm{deg}^{-1}$ to the flux calculated at opposition. The STM has clear limitations with respect to flux accuracy (e.g. Müller \& Blommaert 2004) or for modelling of minor bodies outside the main belt (e.g. Harris 1998), but highly accurate flux predictions are not crucial for our goals.

\subsection{Number counts, fluctuation power and confusion noise}

Number counts: Two kinds of number count quantities are calculated: (1) $N_{\text {tot }}\left(\lambda_{i}\right)$, the total count of asteroids in the counting cell, normalized by the solid angle of the counting cell $\Omega_{\mathrm{c}}\left(\mathrm{sr}^{-1}\right)$; and (2) $N_{\text {lim }}\left(\lambda_{i}, S_{\text {lim }}\right)$, the count of asteroids above the detection limit $S_{\text {lim }}$ in a particular counting cell, normalized by the solid angle of the counting cell $\Omega_{\mathrm{c}}\left(\mathrm{sr}^{-1}\right)$.

Fluctuation powers: The full fluctuation power (see Lagache et al. 2003, for an introduction) is calculated from the "observed" distribution of all asteroids in that specific cell, for a specific $\lambda_{i}$ wavelength:

$\delta F_{0}\left(\lambda_{i}\right)=\left(\frac{1}{\Omega_{\mathrm{c}}}\right) \sum_{j} S_{j}^{2}\left(\lambda_{i}\right)$.

In this case the summation runs over all of the asteroids in the counting cell $\left(\mathrm{sr}^{-1}\right)$.

The fluctuation power due to non-detectable asteroids, $\delta F_{\text {lim }}$, can be calculated for a specific instrument in a similar way as $\delta F_{0}$, but in this case only asteroids below the detection limit $S_{\lim }$ are considered:

$\delta F_{\lim }\left(\lambda_{i}, S_{\lim }\right)=\left(\frac{1}{\Omega_{\mathrm{c}}}\right) \sum_{S_{j}<S_{\lim }} S_{j}^{2}\left(\lambda_{i}\right)$.

Note that $\delta F_{\lim }$ is instrument-dependent only through the actual sensitivity limit, and other characteristics of the instrument, like spatial resolution, are not taken into account in the calculation of $\delta F_{\text {lim. }}$. These properties are considered in the calculation of the confusion noise. $\delta F_{0}$ is a fully instrument-independent quantity, and both $\delta F_{0}$ and $\delta F_{\text {lim }}$ depend strongly on the actual asteroid model.

Confusion noise: Throughout this paper we assume that the local spatial distribution of the asteroids is Poissonian, i.e. the same fluctuation power can be used to calculate the confusion 
noise at any spatial frequency, independent of the instrument. Thus confusion noise can be calculated from the fluctuation powers as:

$\sigma_{0}\left(\lambda_{i}, \Omega_{\mathrm{p}}\right)=\left(\Omega_{\mathrm{p}} \cdot \delta F_{0}\left(\lambda_{i}\right)\right)^{\frac{1}{2}}$

$\sigma_{\lim }\left(\lambda_{i}, S_{\lim }, \Omega_{\mathrm{p}}\right)=\left(\Omega_{\mathrm{p}} \cdot \delta F_{\lim }\left(\lambda_{i}, S_{\lim }\right)\right)^{\frac{1}{2}}$

for the "full" and sensitivity limit dependent confusion noise values, respectively. $\Omega_{\mathrm{p}}$ is the effective solid angle of the detector, which is not necessarily the physical size of the actual pixel/aperture, and the confusion noise applicable for detection limits of point sources depends on the point source flux extraction/reconstruction method as well, (see e.g. Kiss et al. 2005). "Best estimates" of effective solid angles of various instruments and filters can be found at our webpage ${ }^{4}$.

Due to their relatively low number density, asteroids in our model limit the detectability of point sources through the photometric, rather than the number density limit (see Lagache et al. 2003, for a detailed introduction). $\sigma_{0}$ and $\sigma_{\lim }$ are lower limits, since there is an unknown contribution of small (fainter) asteroids, which is not considered here (see Sect. 4 for a discussion).

In the following, unless otherwise quoted, we refer to all these quantities as specific for a given wavelength or photometric band and instrument, and therefore the wavelength, sensitivity limit and spatial resolution (effective detector solid angle) dependences are not marked.

\section{Results}

\subsection{General results}

The positions of the asteroids in the original SAM model were integrated in the time span January 1, 2000 to December 31, 2012. At each date a spectral energy distribution was assigned to each asteroid using the STM, as described in Sect. 2.2. Fluctuation powers and number counts have been derived from this database, which is publicly available at the URL: "http://kisag.konkoly.hu/ solarsystem/irsam.html". The web-interface is described in more detail in Appendix A.

For some specific instruments and time spans we constructed $\delta F_{0}, \delta F_{\text {lim }}, N_{\text {tot }}$, and $N_{\text {lim }}$ maps to characterize the impact of main belt asteroids for the selected instruments. An example is shown in Fig. 2.

The fluctuation power and number count maps at different wavelengths (and the same date) show a very similar morphology. Considering the celestial structure, the main characteristics are the following (presented in the ecliptic coordinate system):

- The $\delta F_{0}$ and $N_{\text {tot }}$ distributions (and so $\delta F_{\text {lim }}$ and $N_{\text {lim }}$ ) are symmetric in ecliptic latitude $(\beta)$, and show a maximum at the ecliptic plane at a specific ecliptic longitude $(\lambda) ; \delta F_{0}$, $\delta F_{\text {lim }}, N_{\text {tot }}$ and $N_{\text {lim }}$ show a similar morphology in celestial distribution.

- The maximum extension in $\beta$ is at the anti-solar point, where fluctuation power isocontours form a "bulge", while the minimum is at the celestial position of the Sun. When comparing different dates, the bulge around the anti-solar point moves along the ecliptic as Earth revolves around the Sun, and so does the minimum.

\footnotetext{
${ }^{4}$ http://kisag.konkoly.hu/solarsystem/irsam.html
}

- If maps presented in the ecliptic coordinate systems ( $\lambda-\beta$ maps) are transformed to helioecliptic coordinate system $\left(\left[\lambda-\lambda_{\odot}\right]-\beta\right.$, where $\lambda_{\odot}$ is the ecliptic longitude of the Sun), then the maps of different dates are rather similar. The differences are about one order lower than the median values of the different maps. Therefore it is possible to create time-independent average maps for a specific instrument setup, which can give "average" fluctuation power and number count estimates, without the need to specify the exact day of the observation.

The main component of the asteroid fluctuation power (or confusion noise) as well as the number counts can be well represented by a map, which is constant in the helioecliptic coordinate system. This kind of map can serve as a good guideline to characterize the expected impact of main belt asteroids for the infrared and submillimetre measurements of a specific instrument, if the exact date of the observation is not known. We present these maps for a handful of instruments in Appendix B, and they are also available in FITS format at our webpage ${ }^{4}$.

For medium ecliptic latitudes the asteroidal sky changes in a timescale of a few weeks, with an actual value depending on the instrument (wavelength and sensitivity limit) and sky position. Close to the ecliptic plane the amplitude of these temporal changes are less pronounced, and there are practically no changes at high ecliptic latitudes since asteroids are present at these places only sporadically.

\subsection{The importance of main belt asteroids for specific instruments}

Asteroids may affect IR and submillimetre observations in two ways: they can increase the fluctuation power (and hence the confusion noise) level, and contribute to the number count of point sources. In our investigated wavelength regime the main sources of confusion noise are the extragalactic background and the Galactic cirrus emission. The strength of the cirrus emission and confusion noise changes rapidly from place to place in the sky and is below the extragalatic confusion noise level in the best "cosmological" windows for most of the space IR instruments (see e.g. Kiss et al. 2005).

Being constant and present in any direction, extragalactic background fluctuations represent a minimum value for the confusion noise. Therefore we used the respective extragalactic fluctuation powers to judge, whether asteroid confusion noise has to be considered for a specific instrument. In the calculations presented in Table 1, we considered an instrument as "affected", if the asteroid confusion noise level was at least half of the extragalactic background component (as calculated by Lagache et al. 2003, 2004).

In the case of infrared space instruments there is always a solar elongation constraint for the actually observable part of the sky, e.g. $60^{\circ} \leq \lambda-\lambda_{\odot} \leq 120^{\circ}$ for the Herschel Space Telescope, $85^{\circ} \leq \lambda-\lambda_{\odot} \leq 120^{\circ}$ for the Spitzer Space Telescope and $89^{\circ} \leq \lambda-$ $\lambda_{\odot} \leq 91^{\circ}$ for Akari. Instruments of these spacecrafts can never look at or close to the anti-solar point, where the highest asteroid fluctuation power is expected.

As a general result of the comparison of different wavelengths, instruments working in the far-infrared regime are not affected, while instrument working in the $5 \mu \mathrm{m} \leq \lambda_{i} \leq 30 \mu \mathrm{m}$ domain can be severely affected by asteroid confusion noise, at least along or close to the ecliptic. Although far-infrared instruments are not affected by the asteroid confusion noise, asteroids above the detection limit may have a considerable impact on the 
Table 1. Comparison of the extragalactic and asteroid full fluctuation power levels at some representative wavelengths of infrared and submillimetre space instruments. Asteroid fluctuation power was derived at the specified $\lambda-\lambda_{\odot}$ helioecliptic longitude, and at the ecliptic plane. The columns of the table are: 1) name of the instrument; 2) wavelength; 3) extragalactic background fluctuation power (EGB); 4) asteroid fluctuation power (MBA); 5) solar elongation used in the MBA fluctuation power calibration, in accordance with the visibility constraints of the instrument; 6) Ecliptic latitude range above/below the ecliptic plane, where a specific instrument is considered as "affected" by asteroid confusion noise. Missing values indicate that the instrument is not affected.

\begin{tabular}{lrrrrc}
\hline \hline Instrument & $\begin{array}{r}\lambda_{i} \\
(\mu \mathrm{m})\end{array}$ & $\begin{array}{r}\log _{10}\left(\delta F_{0} / \mathrm{Jy}^{2} \mathrm{sr}^{-1}\right) \\
(\mathrm{EGB})\end{array}$ & $\begin{array}{r}\lambda-\lambda_{\odot} \\
(\mathrm{MBA})\end{array}$ & $\begin{array}{r}|\Delta \beta| \\
(\mathrm{deg})\end{array}$ \\
\hline Spitzer/IRAC & 8 & 1.49 & 2.11 & 120 & 20 \\
Akari/IRC & 9 & 1.47 & 1.21 & 90 & 13 \\
Akari/IRC & 18 & 1.39 & 2.23 & 90 & 16 \\
Spitzer/MIPS & 24 & 2.04 & 2.70 & 120 & 21 \\
\hline Akari/FIS & 65 & 3.47 & 1.34 & 90 & - \\
Spitzer/MIPS \& Herschel/PACS & 70 & 3.69 & 1.62 & 120 & - \\
Akair/FIS & 90 & 4.06 & 0.98 & 90 & - \\
Herschel/PACS & 100 & 4.42 & 0.95 & 120 & - \\
Akari/FIS & 140 & 4.42 & 0.41 & 90 & - \\
Akari/FIS & 160 & 4.43 & 0.23 & 90 & - \\
Spitzer/MIPS \& Herschel/PACS & 160 & 4.43 & 0.36 & 120 & - \\
Herschel/SPIRE & 250 & 4.29 & -0.21 & 120 & - \\
Herschel/SPIRE & 350 & 3.88 & -0.59 & 120 & - \\
Herschel/SPIRE & 500 & 3.27 & -1.64 & 120 & - \\
\hline
\end{tabular}

source counts. This contribution can be best estimated for a specific measurement (instrument, date, sky position) by the webinterface of our infrared asteroid model.

\section{Discussion}

The SAM is limited to asteroids with a lower limit in size of $1 \mathrm{~km}$ in diameter. There is, certainly, a population of asteroids with sizes below this limit. Since the confusion noise is calculated using Eq. (2), the impact of very small boides (a few hundred meters in diameter and below) is minor to the confusion noise.

To test the effect of small $(D<1 \mathrm{~km})$ asteroids on the fluctuation power and confusion noise we first created a sizefrequency distribution; for asteroids of $D>1 \mathrm{~km}$ we used the number count values estimated by Eq. (3) in Tedesco et al. (2005), while for $D \leq 1 \mathrm{~km}$ the interpolated values of Belton et al. (1992) were used. This SFD is a combination of the "SAM" and "Galileo team" data points in Fig. 4 in Tedesco et al. (2005). A simple relationship of $S\left(\lambda_{i}\right) \propto D^{2}$ was assumed between the flux density of an asteroid's thermal emission at a specific wavelength $S\left(\lambda_{i}\right)$ and its diameter $D$. Then the specific fluctuation power $\delta F(D)$ was calculated for a $D \pm \Delta D$ interval, using Eq. (1). It is clear from Fig. 1 that the fluctuation power is dominated by the bright and large asteroids, and that small $(D \leq 1 \mathrm{~km})$ minor planets have a negligible contribution to the fluctuation power. Presently orbits for almost 400000 asteroids are known, i.e., more than $20 \%$ of the $1.9 \times 10^{6} \mathrm{SAM}$ asteroid sample (see Sect. 1), but only about $0.1 \%$ ( 2000 asteroids) have known sizes and albedos which are the crucial parameters for the confusion noise estimates. The calculated power fluctuations are therefore dominated by asteroids which have been extrapolated in the SAM via size-frequency distributions. However, knowing only $0.1 \%$ is far from being statistically relevant and in some parts of the sky bright (i.e. large) asteroids are completely missing. This implies that confusion noise estimates cannot rely on the known asteroid sample alone and the application of the SAM model for confusion noise calculations is necessary.

On the other hand, fluctuation power and confusion noise are dominated by the largest/brightest asteroids in the actual field,

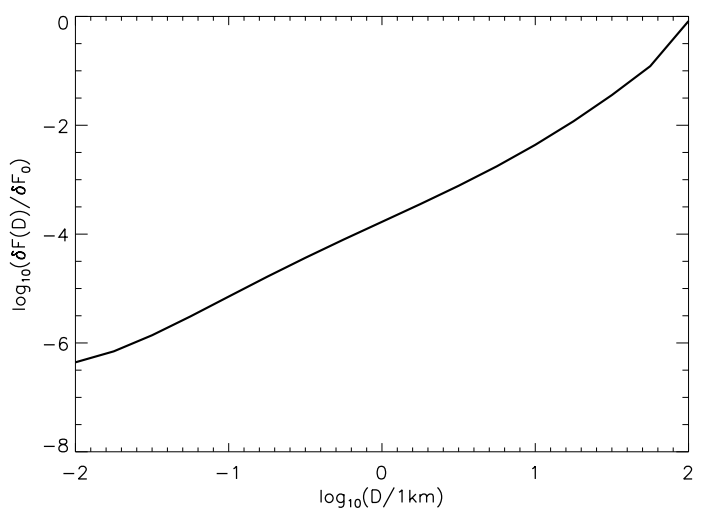

Fig. 1. Relative contribution of asteroids with a specific size $D$ to the asteroid fluctuation power, using the size distribution and the simple flux model as described in Sect. 4.

even if the known ones are missing. As suggested by Fig. 1 the contribution of $D \leq 1 \mathrm{~km}$ asteroids remains negligible, and a further extension of the SAM to smaller diameters would not improve the accuracy of the confusion noise calculations significantly.

Asteroids further out in the Solar System (e.g. transNeptunian objects) will not contribute considerably to the confusion noise or number counts, as deduced from their currently known size distribution in Appendix C.

As discussed in detail in Appendix D, SAM asteroids contribute to the absolute brightness of the zodiacal emission in a negligible level, however, a set of these asteroids may be the dominant source of zodiacal emission fluctuation power at some specific wavelengths and sky regions.

Acknowledgements. This research was supported by the European Space Agency (ESA) and by the Hungarian Space Office via the PECS programme (contract No. 98011). Cs.K. and P.Á. acknowledge the support of the Hungarian Reserch Fund (OTKA K62304). 


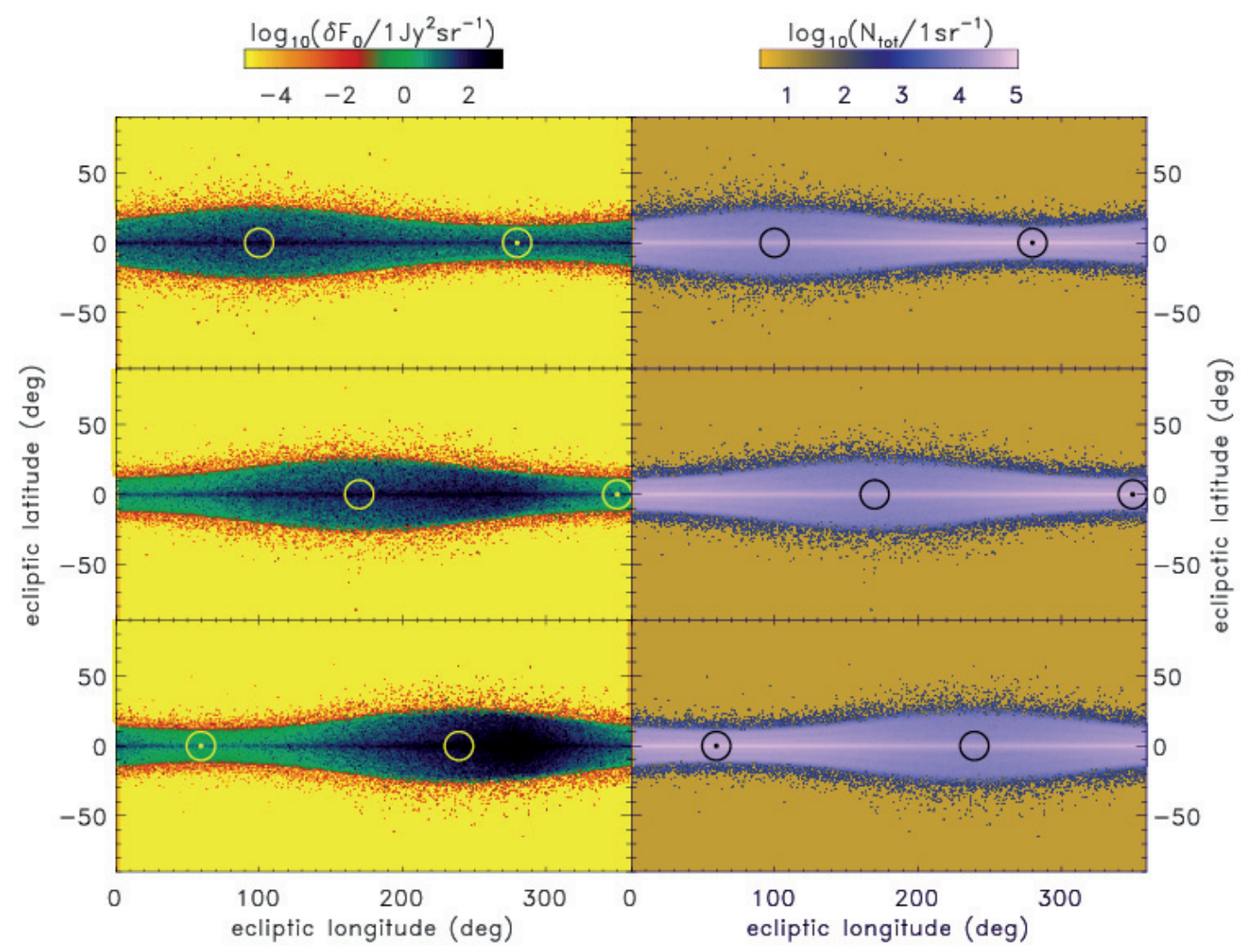

Fig. 2. Expected distribution of fluctuation power $\left(\delta F_{0}\right.$, left column $)$ and the total number of asteroids $\left(N_{\text {tot }}\right.$, right column) over the sky of SAM asteroids at $20 \mu \mathrm{m}$ on - from top to bottom - January 1, March 10 and May 20, 2008 (ecliptic coordinate system). The position of the solar and anti-solar points are marked with "๑" and "○" symbols, respectively.

\section{Appendix A: Web-interface for infrared and submillimetre asteroid fluctuation power and number count estimates}

The results of our model are available through a webinterface, and that can be used to characterize the impact of MBAs on the photometry and source counts, for a specific observational setup. The tool is available at the URL: "http: //kisag. konkoly.hu/solarsystem/irsam.html". The main features of the tool are described below.

The input parameters are the following:

- Right ascension (RA) and declination (Dec) of the target field. Alternatively, one can specify ecliptic coordinates $(\lambda$ and $\beta$ ) as well.

- Date of the observation. In our model the calculations were performed for the period from January 1, 2000 to December 31, 2012. It is possible to give a date outside this range, but then the returned values are calculated for the same solar elongation configuration in the time span above, closest to the supplied date.

- Wavelength of the observation $\left(\lambda_{i}, \mu \mathrm{m}\right)$.

- Sensitivity limit ( $S_{\text {lim }}$, Jy). The fluctuation level due to unresolved sources $\delta F_{\text {lim }}$ and the count of "detectable" asteroids $N_{\lim }=N\left(S>S_{\text {lim }}\right)$ are both calculated respective to $S_{\text {lim }}$.

- Centre of the coordinate system. It is possible to specify a geocentric coordinate system (default), or that of centred at the position of the Spitzer Space Telescope on the supplied date.
The outputs of the form are the following for each individual input parameter (coordinates/date/wavelength/sensitivity) setup:

- $\delta F_{0}$ : Full fluctuation power due to asteroids $\left(\mathrm{Jy}^{2} \mathrm{Sr}^{-1}\right)$.

- $\delta F_{\text {lim }}$ : Fluctuation level due to asteroids below the detection $\operatorname{limit} S_{\lim }\left(\mathrm{Jy}^{2} \mathrm{sr}^{-1}\right)$.

- $N_{\text {lim }}$ : Number of asteroids with fluxes above $S_{\text {lim }}$, at the wavelength specified $\left(\mathrm{sr}^{-1}\right)$.

- $B_{0}$ : Average contribution of asteroids in the SAM model to the background surface brightness, at the wavelength specified $\left(\mathrm{MJy} \mathrm{sr}^{-1}\right)$.

Confusion noise levels for specific instruments can be calculated from the fluctuation powers in the way described in Sect. 2.3.

\section{Appendix B: Mission-average maps for the central wavelengths of current and future space IR instruments}

The major component of fluctuation power due to main belt asteroids is dependent on the solar elongation and the ecliptic latitude of the current target field only, but not on the actual date of the observation (see Sect. 3.1). This component can be well represented by the median values of the fluctuation powers in solar elongation and ecliptic latitude over a longer period, at a specific wavelength. The time spans covered the expected lifetime of space instruments, therefore we called these "median" maps "mission-average".

Here we present mission-average fluctuation power maps for the central wavelengths of the main mid-IR photometric 


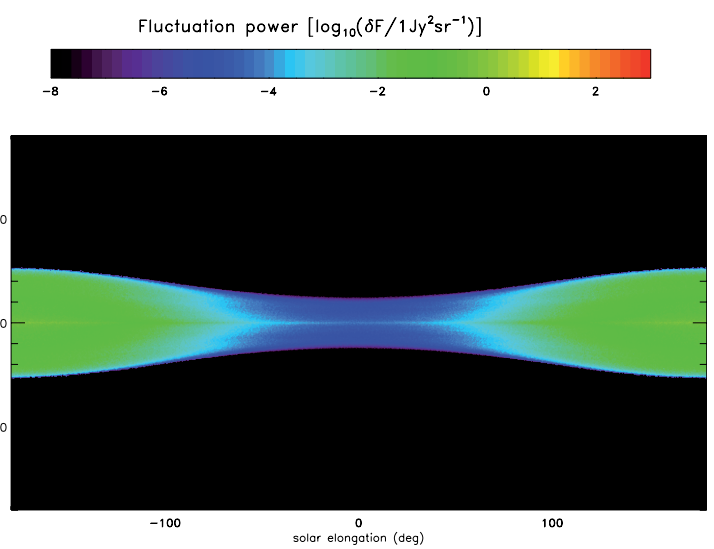

Fig. B.1. Fluctuation power due to asteroids, $\lambda=5 \mu \mathrm{m}$ (general $M$-band).

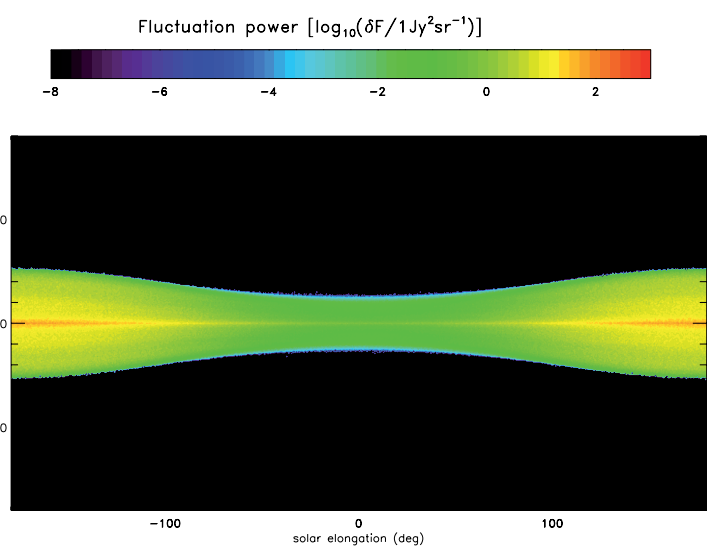

Fig. B.2. Fluctuation power due to asteroids, $\lambda=9 \mu \mathrm{m}$ (Akari/S9W).

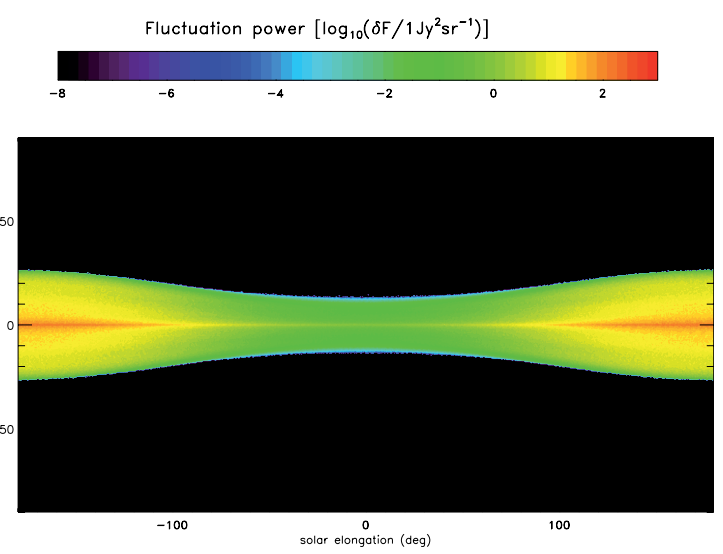

Fig. B.3. Fluctuation power due to asteroids, $\lambda=10 \mu \mathrm{m}$ (general $N$-band).

bands $(M, N$ and $Q)$ and for the central wavelengths of current and future space IR instruments. Mission-average fluctuation power maps are presented for the following instruments/filters:

- General mid-IR photometry in $M, N$ and $Q$ bands (approximately 5, 10 and $19 \mu \mathrm{m})$.

- Akari (Murakami et al. 2007): 9, 18, 65, 90, 140 and $160 \mu \mathrm{m}$.

- Spitzer (Werner et al. 2004): 8, 24, 70 and $160 \mu \mathrm{m}$.

- Herschel/PACS \& SPIRE (Pilbratt 2004): 75, 110, 175, 250, 360 and $520 \mu \mathrm{m}$.

- Planck/HFI (Tauber 2004): 350, 550 and $850 \mu \mathrm{m}$.
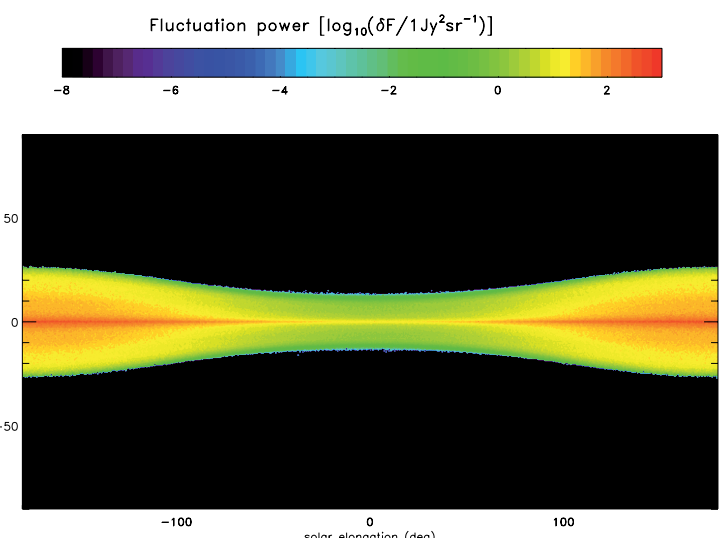

Fig. B.4. Fluctuation power due to asteroids, $\lambda=18 \mu \mathrm{m}$ (Akari/IRC).

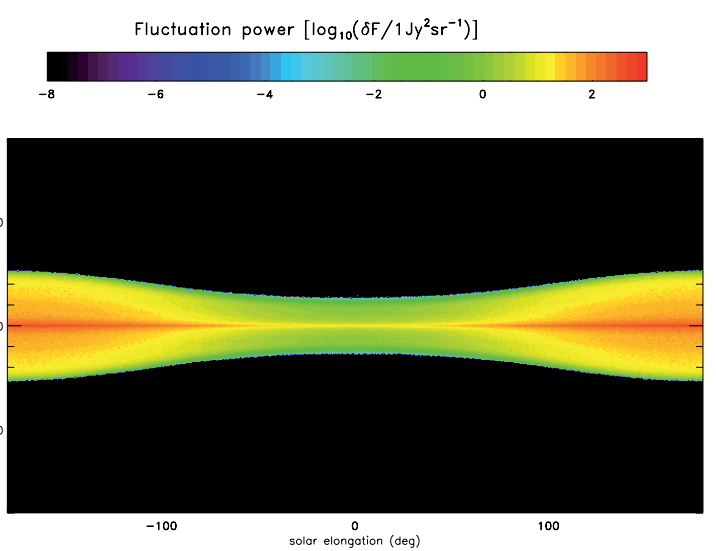

Fig. B.5. Fluctuation power due to asteroids, $\lambda=19 \mu \mathrm{m}$ (general $Q$-band).

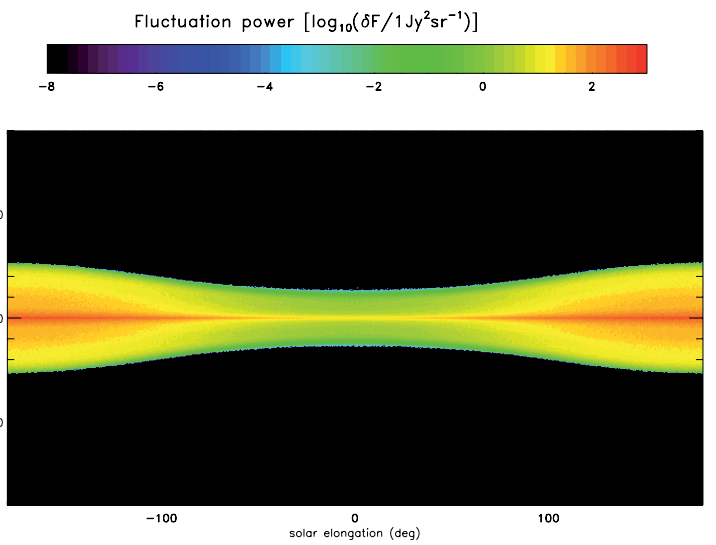

Fig. B.6. Fluctuation power due to asteroids, $\lambda=24 \mu \mathrm{m}$ (Spitzer/MIPS).

The maps presented here are also available in FITS format at the following web address: http://kisag.konkoly.hu/ solarsystem/irsam.html

\section{Appendix C: Cold population}

In our model we considered the asteroid families found in the SAM only, i.e. our asteroids are main belt asteroids, with temperatures typically in the order of a few hundred kelvins. With these temperatures, most of their heat is emitted at mid-IR wavelengths. Asteroids further out in the Solar System (e.g. in the Kuiper belt) have lower temperatures, therefore contribute more 

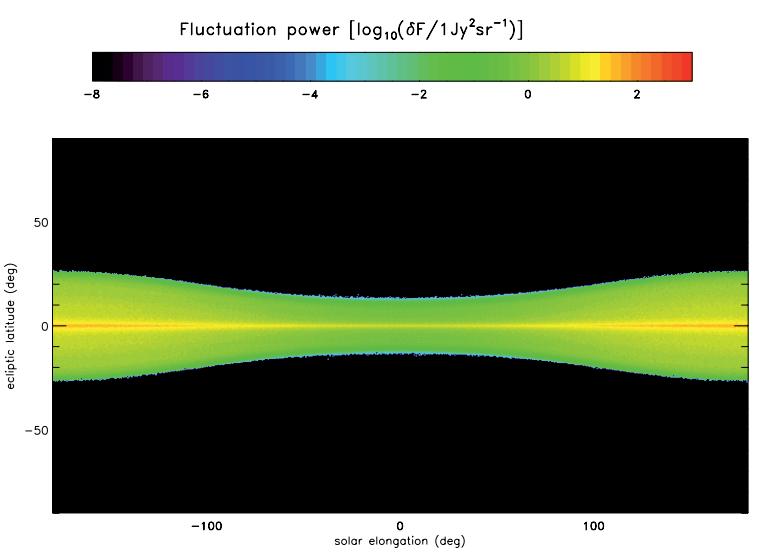

Fig. B.7. Fluctuation power due to asteroids, $\lambda=65 \mu \mathrm{m}$ (Akari/FIS/ N60).

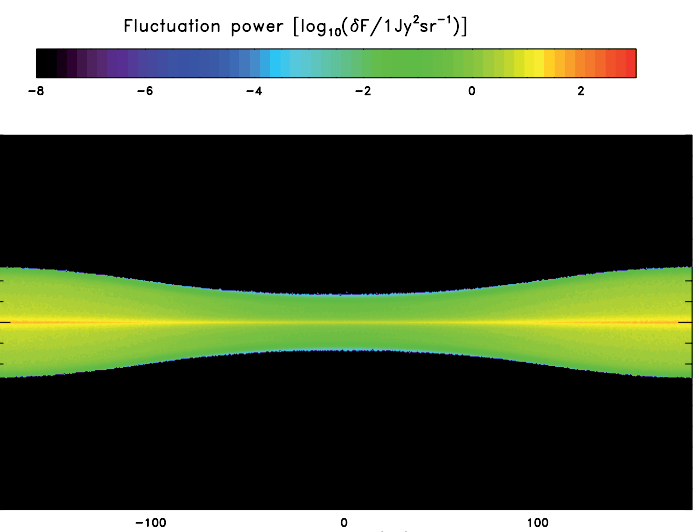

Fig. B.8. Fluctuation power due to asteroids, $\lambda=70 \mu \mathrm{m}$ (Spitzer/MIPS, Herschel/PACS).

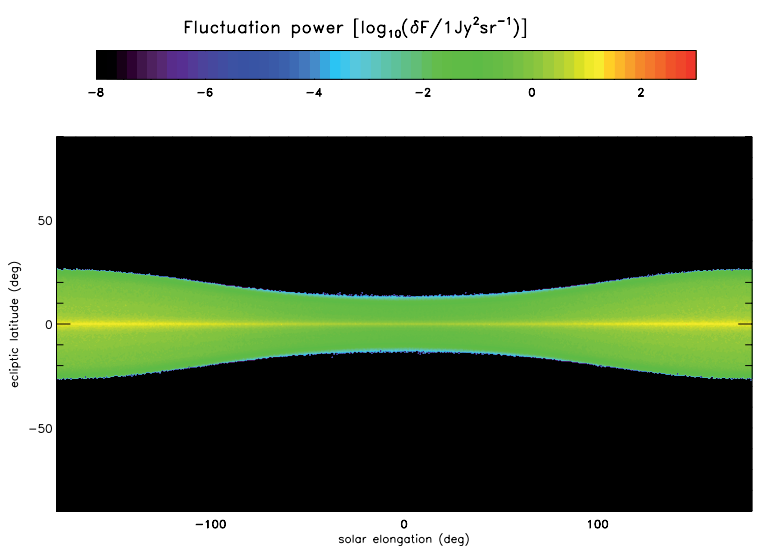

Fig. B.9. Fluctuation power due to asteroids, $\lambda=90 \mu \mathrm{m}$ (Akari/FIS/ WIDE-S).

to the far-infrared part of their spectrum. This effect cannot compensate the fast drop in the observed IR flux due to the larger distance between the asteroid and the observer, and the larger distance of the asteroid to the Sun. We demonstrated this effect in Fig. C.1 where the spectral energy distribution of the same asteroid is plotted, if placed at different distances from the observer. The observed fluxes at at longer wavelengths decrease fast (peak values decrease with increasing distance from the observer/Sun in Fig. C.1) and the displacement of the peak in wavelength (due to the lower body temperatures) cannot compensate that effect. An extreme population of relatively large bodies would be

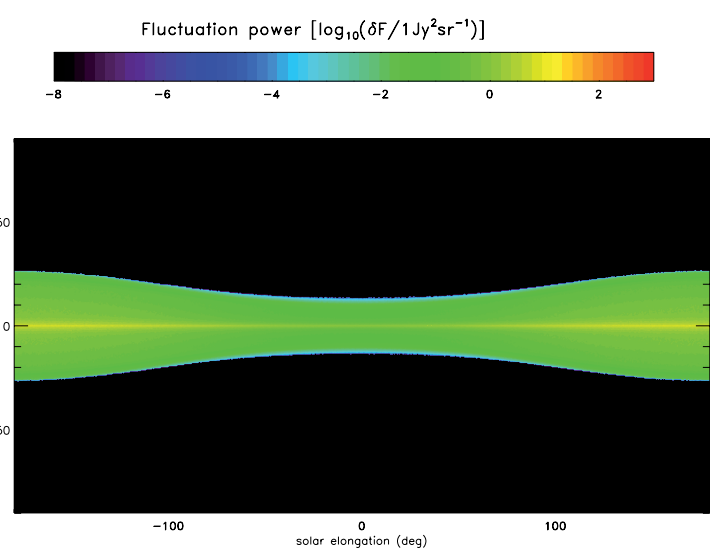

Fig. B.10. Fluctuation power due to asteroids, $\lambda=100 \mu \mathrm{m}$ (Herschel/ PACS).

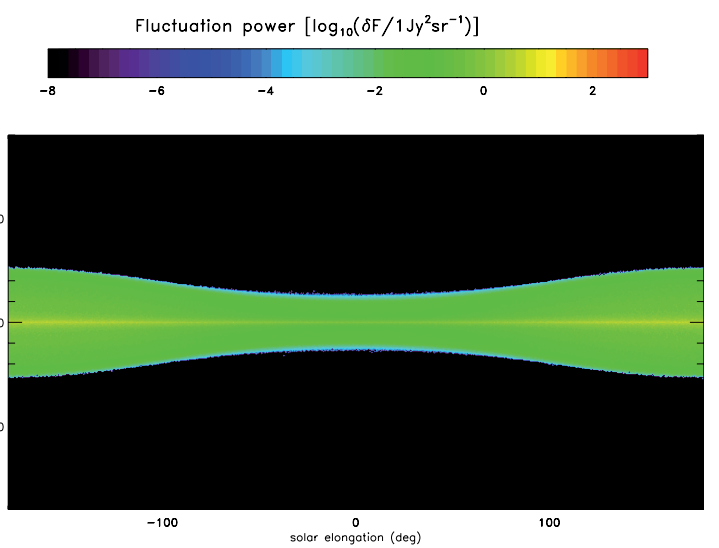

Fig. B.11. Fluctuation power due to asteroids, $\lambda=140 \mu \mathrm{m}$ (Akari/FIS/ WIDE-L).

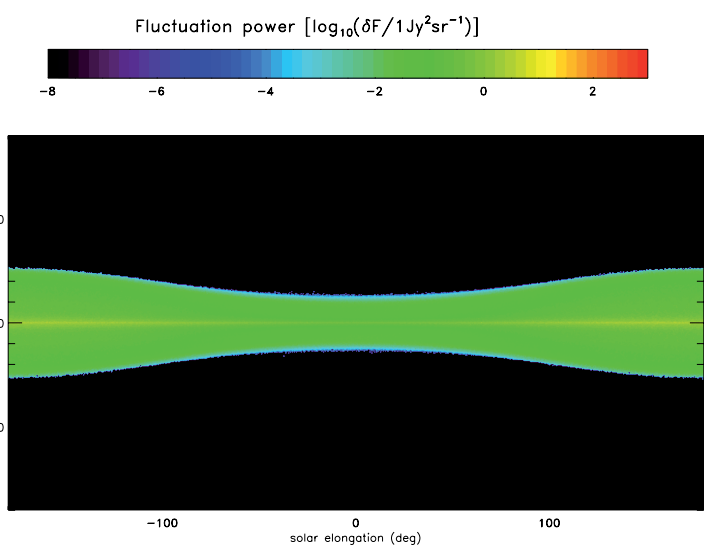

Fig. B.12. Fluctuation power due to asteroids, $\lambda=160 \mu \mathrm{m}$ (Akari/FIS/ N160, Spitzer/MIPS, Herschel/PACS).

needed to have an effect comparable to that of main belt asteroids on the FIR confusion noise and expected count of asteroids, which is not supported by the current number count estimates (Kenyon \& Bromley 2004; Roques et al. 2006).

\section{Appendix D: Contribution to the zodiacal emission}

The asteroids of our model contribute to the zodiacal emission in two ways: to the absolute sky brightness and to the fluctuations. The contribution of SAM asteroids to the surface brightness is negligible, on average it is in the $10^{-3}-10^{-8}$ level to the total 


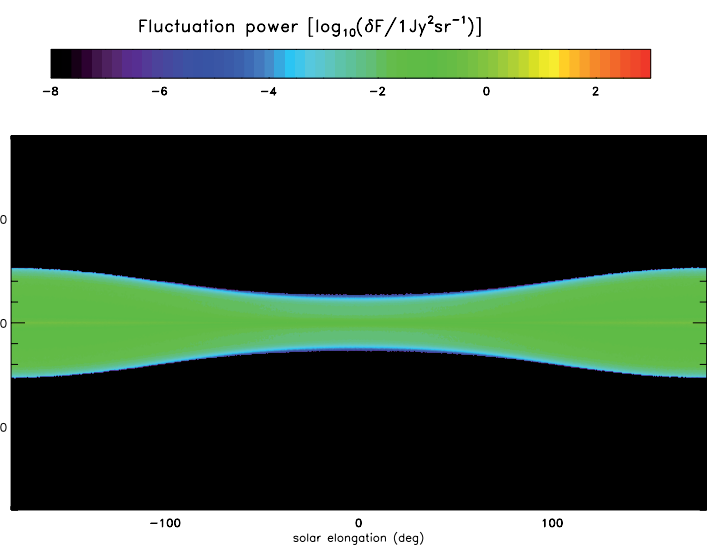

Fig. B.13. Fluctuation power due to asteroids, $\lambda=250 \mu \mathrm{m}$ (Herschel/ SPIRE).

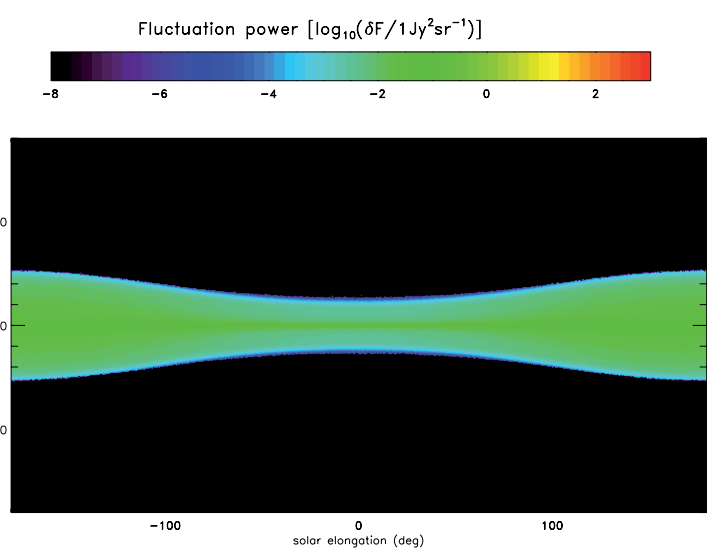

Fig. B.14. Fluctuation power due to asteroids, $\lambda=350 \mu \mathrm{m}$ (Planck/HFI, Herschel/SPIRE).

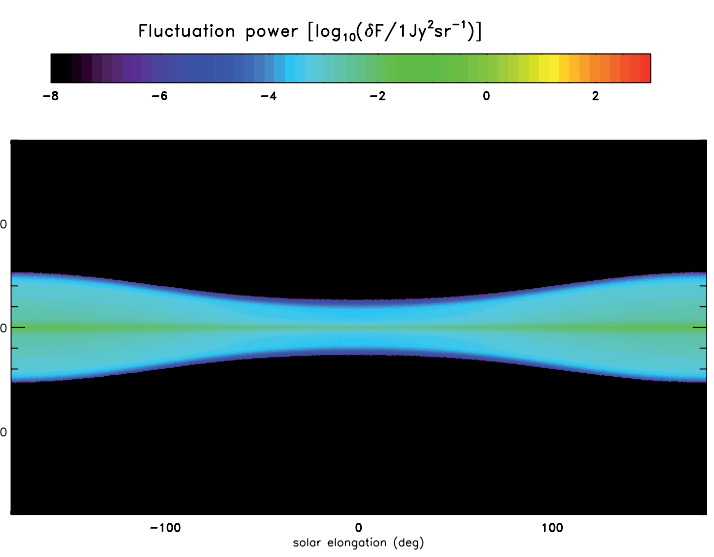

Fig. B.15. Fluctuation power due to asteroids, $\lambda=500 \mu \mathrm{m}$ (Herschel/ SPIRE).

brightness of the zodiacal emission. Since the average spectral energy distribution of the SAM asteroids and the zodiacal emission are rather similar, this ratio does not change remarkably with wavelength.

SAM asteroids contribute more significantly to the smallscale surface brightness flucutations in the zodiacal emission, which is currently best known from ISOPHOT observations. Ábrahám et al. (1997) found an upper limit of $0.2 \%$ of the total brightness for the fluctuation amplitude of the small-scale zodiacal emission fluctuations at $25 \mu \mathrm{m}$. To compare these limits with

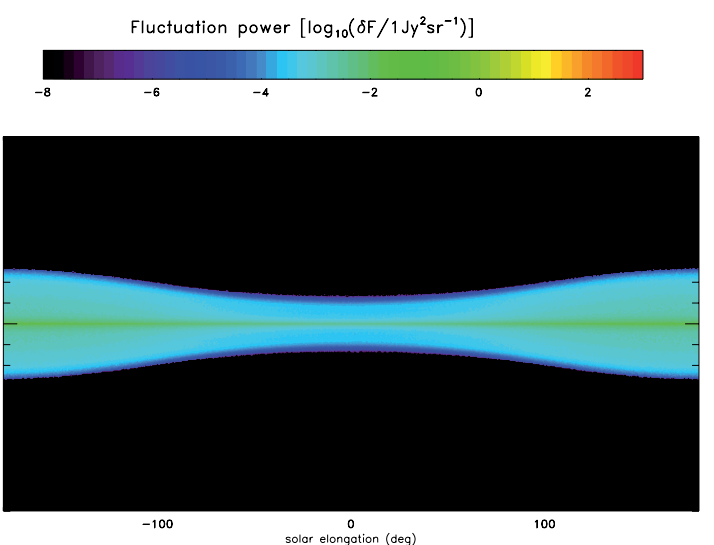

Fig. B.16. Fluctuation power due to asteroids, $\lambda=550 \mu \mathrm{m}$ (Planck/HFI).

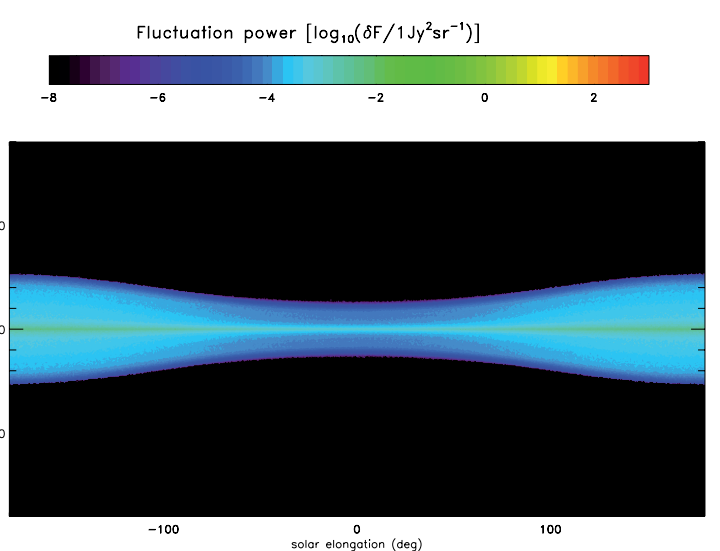

Fig. B.17. Fluctuation power due to asteroids, $\lambda=850 \mu \mathrm{m}$ (Planck/HFI).

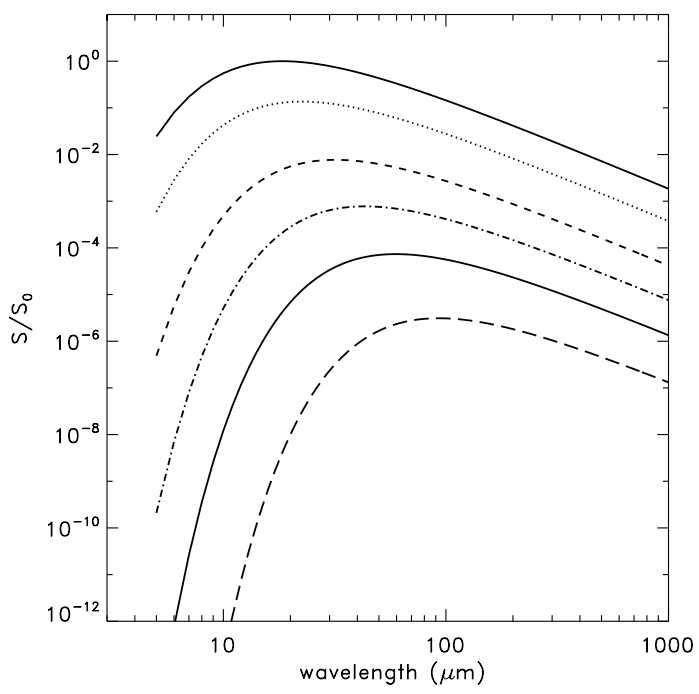

Fig. C.1. Demonstration of the effect of the increasing geocentric and heliocentric distances on the observed fluxes of the asteroids. $S / S_{0}$ is the ratio of measured $S$ flux value at a specific wavelength and the reference flux value $S_{0}$ (the maximum value of the spectral energy distribution at $\Delta=1 \mathrm{AU}$ ). The curves correspond to $\Delta=1,2,5,10,20$ and $50 \mathrm{AU}$, from top to bottom, respectively and $r_{\odot}=\Delta+1 \mathrm{AU}$ in all cases.

the fluctuation power due to SAM asteroids, we transformed the surface brightness of the zodiacal emission component $B_{\mathrm{ZE}}$, estimated by the PredictDIRBE tool (see Appendix A in Kiss et al. 2006), to fluctuation power limits $\mathrm{d} F_{\mathrm{U}}$ as $\delta F_{\mathrm{U}}=\delta B_{\mathrm{ZE}}^{2} \times \Omega_{\mathrm{PHT}}$, where $\delta B_{\mathrm{ZE}}=0.002 \cdot B_{\mathrm{ZE}}$, the upper limit of the surface 
brightness fluctuation amplitude, and $\Omega_{\mathrm{PHT}}$ is the solid angle corresponding to the $3^{\prime}$ aperture used in Ábrahám et al. (1997). These $\delta F_{\mathrm{U}}$ values can be directly compared to the actual $\delta F_{0}$ values of our model. We performed this comparison in the vicinity of the ecliptic, for some sky positions covered by the Spitzer First Look Survey Ecliptic Plane Component (FLS EPC, Meadows et al. 2004). The ratio of the SAM fluctuation powers to the upper limits derived from the zodiacal emission surface brightness $\delta F_{0} / \delta F_{\mathrm{U}}$ varies in the range $0.05 \leq \delta F_{0} / \delta F_{\mathrm{U}} \leq$ 0.15 , depending mainly on the ecliptic latitude, and decreases slowly (by a few per cent) from $\beta=0^{\circ}$ to $|\beta| \approx 10^{\circ}$, as we move further from the ecliptic at a constant $\lambda$.

As we have seen, the asteroid fluctuation powers were well below the detection limit of ISOPHOT, if these were the only source of the zodiacal fluctuation power. However, we checked, whether these fluctuation powers are achievable by the present infrared instrumentation, especially with the Spitzer Space Telescope. Accoring the Meadows et al. (2004) the Spitzer FLS EPC had $0.08 \mathrm{mJy}$ and $0.8 \mathrm{mJy}$ sensitivity limits for the IRAC $8 \mu \mathrm{m}$ (Fazio et al. 2004) and MIPS $24 \mu \mathrm{m}$ (Rieke et al. 2004) instruments/filters respectively. These correspond to $\delta S_{8} \approx 100 \mathrm{Jy}^{2} \mathrm{sr}^{-1}$ and $\delta S_{24} \approx 1000 \mathrm{Jy}^{2} \mathrm{sr}^{-1}$ fluctuation powers on the spatial scales of the respective detector pixels ${ }^{5}$. The fluctuation powers due to SAM asteroids varies in the ranges $30 \ldots 80 \mathrm{Jy}^{2} \mathrm{sr}^{-1}$ and $500 \ldots 1000 \mathrm{Jy}^{2} \mathrm{sr}^{-1}$ for 8 and $24 \mu \mathrm{m}$, respectively, in the selected FLS EPC positions. This shows that asteroid fluctuation power is an important source of confusion noise for these instruments in these parts of the sky and in some cases is the dominant source of confusion, also limiting the local sensitivity of point source flux determination. A detailed analysis of the fluctuations on FLS EPC maps or similar datasets is necessary to characterize the impact of asteroids on the zodiacal emission at different ecliptic latitudes and to disentangle its small-scale brightness fluctuations from the noise sources ${ }^{6}$.

\section{References}

Ábrahám, P., Leinert, Ch., \& Lemke, D. 1997, A\&A, 328, 702

Belton, M. J. S., Veverka, J., Thomas, P., et al. 1992, Science, 257, 1647

Fazio, G. G., Hora, J. L., Allen, L. E., et al. 2004, ApJS, 154, 10

Harris, A. W. 1998, Icarus, 131, 291

Jeong, W. S., Lee, H. M., Pak, S., et al. 2005, MNRAS, 357, 535

Jeong, W. S., Pearson, C. P., Lee, H. M., Pak, S., \& Nakagawa, T. 2006, MNRAS, 369,281

Kiss, Cs., Klaas, U., \& Lemke, D. 2005, A\&A, 430, 343

Kiss, Cs., Ábrahám, P., Laureijs, R. J., Moór, A., \& Birkmann, S. M. 2006, MNRAS, 373, 1213

Kenyon, S. J., \& Bromley, B. C. 2004, AJ, 128, 1916

Lagache, G., Dole, H., \& Puget, J.-L. 2003, MNRAS, 338, 555

Lagache, G., Dole, H., Puget, J.-L., et al. 2004, ApJS, 154, 112

Lebofsky, L. A., Sykes, M. V., Tedesco, E. F., et al. 1986, Icarus, 68, 239

Meadows, V. S., Bhattacharya, B., Reach, W. T., et al. 2004, ApJS, 154, 469

Müller, T. G., \& Blommaert, J. A. D. L. 2004, A\&A, 418, 347

Murakami, H., Baba, H., Barthel, P., et al. 2007, PASJ, in press [arXiv: 0708.1796]

Negrello, M., Magliocchetti, M., Moscardini, L., et al. 2004, MNRAS, 352, 493 Pilbratt, G. L. 2004, ESA-SP, 577, 3

Rieke, G. H., Young, E. T., Engelbracht, C. W., et al. 2004, ApJS, 154, 25

Roques, F., Doressoundiram, A., Dhillon, V., et al. 2006, AJ, 132, 819

Tauber, J. A. 2004, Adv. Space Res., 34, 491

Tedesco, E. F., \& Désert, F.-X. 2002, AJ, 123, 2070

Tedesco, E. F., Cellino, A., \& Zappalá, V. 2005, AJ, 129, 2869

Werner, M. W., Roelling, T. L., Low, F. J., et al. 2004, ApJS, 154, 1

\footnotetext{
${ }^{5}$ Note, that the conversion of photometric uncertainties to fluctuation powers depends on the point source flux reconstruction method and effective solid angle of the measuring apertures, and therefore are accurate in the present case within a factor of $\sim 2$
}

${ }^{6}$ Detailed information on the Spitzer programs are available at the Spitzer Space Telescope website: http://ssc.spitzer.caltech.edu/ 